Rev. Int. Contam. Ambie. 37, 565-576, 2021

https://doi.org/10.20937/RICA.53991

\title{
EVOLUCIÓN DE LOS FACTORES DE EMISIÓN COMO HERRAMIENTA DE MEJORA AMBIENTAL. CASO DE ESTUDIO DEL SECTOR CEMENTERO ESPAÑOL
}

\author{
Evolution of emission factors as an environmental improvement tool. Case study of the Spanish cement sector
}

Pedro MORA, Esther GIL, Laura SÁNCHEZ-MARTÍN y Bernardo LLAMAS*

Escuela Técnica Superior de Ingenieros de Minas y Energía, Universidad Politécnica de Madrid, Rosas 21, 28003 Madrid, España.

*Autor para correspondencia: bernardo.1lamas@upm.es

(Recibido: marzo de 2020; aceptado: octubre de 2020)

Palabras clave: protección ambiental, fabricación de cemento, factores de emisión.

\section{RESUMEN}

La concienciación ambiental en el sector cementero español, unido a la normalización en cuanto a la cuantificación de emisiones nocivas para la salud, seguridad y ambiente, ha propiciado el desarrollo de factores de emisión como herramienta de estandarización a nivel país. Gracias a estos factores es posible establecer su evolución y analizar las causas técnicas que inciden en el comportamiento de las emisiones contaminantes. El presente trabajo de investigación recoge la evolución de dichos factores en una serie histórica que abarca el periodo 2009-2018. El análisis estadístico desarrollado permite establecer factores de emisión para el sector de estudio de forma robusta y fiable. Los resultados recogen la evolución de las emisiones, así como los últimos valores tomados de todo un sector de la industria española. La evolución de los factores de emisión muestra una concienciación cada vez mayor hacia la reducción de gases contaminantes, validando la herramienta factores de emisión y su metodología.

Key words: environmental protection, cement manufacture, emissions factor.

\begin{abstract}
Environmental issues relevant to the Spanish cement sector, together with the regulation of emissions which are harmful to health, safety and the environment, have led to the development of emission factors as a standardization tool at sector level. According to these factors it is possible to establish their evolution and analyze the technical causes that influence the behavior of polluting emissions. This research includes this evolution in a historical series encompassing the period 2009-2018. The statistical analysis developed makes it possible to establish emission factors for the cement sector in a robust and reliable way. The results include the evolution of emissions as well as the latest values taken from an entire industrial sector in Spain. The evolution of emission factors shows an increasing awareness towards the reduction of polluting gases, validating the emission factors tool and its methodology.
\end{abstract}




\section{INTRODUCCIÓN}

El control de la protección del ambiente y la contaminación antrópica producida por las diferentes actividades industriales es uno de los aspectos que más ha evolucionado desde el punto de vista técnico, social y, por ende, legislativo. El enfoque ha evolucionado, pasando de una estrategia correctiva a otra totalmente preventiva cuyo objetivo principal es reducir la contaminación mediante una visión holística de los procesos, protegiendo el medio ambiente de forma global y siendo más transparentes y accesibles en cuanto a información ambiental.

Para un control exhaustivo de la calidad del aire de forma preventiva y anticipada en el tiempo, es necesario estimar las emisiones mediante modelos estadísticos que se ajusten a la realidad de cada entorno, basados en las emisiones de sustancias contaminantes, normalmente simulados mediante factores de emisión (FE) (INECC 2013).

La transparencia y accesibilidad de datos es una cuestión primordial y los registros de emisiones y transferencias de contaminantes son una de las herramientas más adecuadas y utilizadas a nivel global para tener a disposición información y realizar el seguimiento y control de las emisiones (Mora-Peris et al. 2018). Por ello es necesario vigilar y recopilar tanto la emisión de gases contaminantes de $\mathrm{CO}_{2}$, $\mathrm{NO}_{\mathrm{x}}, \mathrm{SO}_{\mathrm{x}}$ (Barba et al. 2006, Zemba et al. 2011), como las de metales pesados, furanos, dioxinas (Rovira et al. 2014) y partículas sólidas, entre las que cabe destacar las $\mathrm{PM}_{10}$ (Pérez Vidal et al. 2010, Abril et al. 2014). Cualquier ciclo de un producto que implique extracción, transporte y otras fases, conduce a la emisión de estos componentes (Barba et al. 2007, Syamala Devi et al. 2017).

El presente estudio tiene como objetivo evaluar la emisión de contaminantes en los procesos de combustión para la obtención de una tonelada de clínker, en los que se consumen entre 3000 y $6500 \mathrm{MJ}$ de energía, dato que varía según el proceso de fabricación y el tipo de producto (García-Gusano et al. 2015), por un lado, y por otro a las mejoras tecnológicas de los hornos (Barba et al. 2006), implementando las mejores tecnologías disponibles (MTD).

En el caso del cemento, los procesos de combustión, como se ha comentado previamente, generan $\mathrm{NO}_{\mathrm{x}}, \mathrm{SO}_{2}$ y otros contaminantes menores (Valderrama et al. 2012), pudiendo incluso considerarse aquellos con contenido de cloro (Carrasco et al. 2010, Laribi et al. 2017). Este tipo de emisiones son características tanto de los hornos húmedos (Zimwara et al. 2012) como los de vía seca. La mejor solución aplicable a las existentes es la aplicación de las MTD por vía seca (Hoyos-Barreto et al. 2008).

Las partículas sólidas con diámetro de $10 \mu \mathrm{m}$ $\left(\mathrm{PM}_{10}\right)$ que pueden tener origen en este tipo de industrias (Pérez-Vidal et al. 2010), contienen algún componente dañino que causa malestares como irritación de garganta y ojos, e incluos enfermedades cardiovasculares, asmáticas o respiratorias crónicas (HEI 2002, Pérez-Vidal et al. 2010). Esto se debe principalmente a su tamaño reducido (Gupta et al. 2012, Sana Mehraj et al. 2013, Rovira et al. 2018), el cual facilita la penetración al tórax por inhalación e influye en la aparición de células cancerígenas, teratogénicas y mutagénicas (Arden et al. 1995, Pérez-Vidal et al. 2010). Diversos estudios han demostrado que los parámetros meteorológicos como precipitación y viento no sólo pueden perjudicar la flora y la fauna (HEI 2000, Pérez-Vidal et al. 2010), sino que además la estacionalidad es otra constante que debe tenerse en cuenta, ya que, por ejemplo, las emisiones son mayores en diciembre y con condiciones anticiclónicas y menores en verano o en la época de lluvia (Pey et al. 2010, Rovira et al. 2018). Por ello la identificación y cuantificación de las fuentes de contaminación por partículas sólidas se han convertido en un tema cada vez más trascendente (Kong et al. 2011, Gupta et al. 2012).

Por otro lado, los contaminantes orgánicos persistentes (COP), caracterizados como dioxinas $\mathrm{y}$ furanos (PCDD-PCDF) son nocivos por sus propiedades lipófilas y sus efectos bioacumulativos persistentes en los seres humanos y el ambiente (van den Berg et al. 2006, Sana-Mehraj et al. 2013); sin embargo, en contraposición con la creencia que ha persistido durante décadas, se ha demostrado que la industria cementera no es uno de los emisores significativos de este tipo de contaminante (Raffetti et al. 2019); es más, contribuye con un porcentaje inferior al $1 \%$ de las emisiones globales (Karstensen 2008). Se ha probado incluso que muchos contaminantes provienen del tráfico de vías de comunicación públicas de la red estatal o regional localizadas en los alrededores de las cementeras (Bertoldi et al. 2012, Rovira et al. 2018). Situación similar ocurre con los metales pesados, cuyas emisiones no superan los límites establecidos por la legislación (DOUE 2010, 2013), y la fuente de emisión en estudio no tiene por qué afectar a la flora, la fauna o el ambiente en general.

El mayor productor de cemento a nivel mundial es China, con casi $2.4 \times 10^{3} \mathrm{Mt}$ en 2018 , seguida por India con $290 \mathrm{Mt}$ en el mismo año (Estatista 2019). España se situó en una producción próxima 
a los $20 \mathrm{Mt}$ en 2019. Para reducir las emisiones de la industria cementera se han combinado la mejora en la eficiencia energética, el uso de sustitutos del clínker (tales como cenizas volantes y escorias [SitiAktar y Haslenda 2015]) y el cambio a combustibles alternativos (McLellan et al. 2012). El sector del cemento es responsable del 12-15\% de la energía primaria consumida (Ali et al. 2011), de la cual el $75 \%$ corresponde al combustible usado en la fase de fabricación (Madlool et al. 2011). Extensos estudios han demostrado que recurrir al uso de combustibles alternativos, tales como aceite de motor, residuos urbanos, residuos de pintura y residuos de tratamiento de efluentes (Gupta et al. 2012, Rovira et al. 2014) permite disminuir las emisiones en una media del $40 \%$ (Hoyos-Barreto et al. 2008).

Debido a esta preocupación ambiental, el cambio de combustible en las fábricas de cemento es una estrategia seguida en muchos países, y su rendimiento individual se evalúa constantemente en función de normativas reglamentarias (Richards y Agranovski 2017). En España, la administración está tomando medidas para limitar en el año 2025 las emisiones antrópicas de $\mathrm{NO}_{\mathrm{x}}, \mathrm{SO}_{2}, \mathrm{COV}$ s no metálicos, amoniaco y partículas finas (BOE 2018).

De ahí que los FE e inventarios de emisión (IE) sean herramientas fundamentales para la gestión de la calidad del aire (Yeo et al. 2016). La estimación de emisiones puede ser la clave para desarrollar, controlar y evaluar las estrategias de emisión, a fin de determinar la aplicación de controles y programas, inventarios de efectos de las fuentes adecuadas y estrategias de mitigación, así como cualquier otro tipo de aplicación relacionada, vinculando al Estado y a distintas administraciones, así como agencias locales, consultorías y la propia industria (Mora-Peris et al. 2008). Los FE destacan las fuentes de emisión, cuyos controles pueden llevar a considerables reducciones de contaminación en las zonas geográficas (Canpolat et al. 2002). Sin embargo, hasta ahora la mayoría de los estudios se han centrado en años específicos (Lei et al. 2011).

El presente trabajo tiene como objetivo evaluar y mostrar los resultados del cálculo de los FE de cada contaminante basados en las mediciones de cada fábrica de cemento según el RD 818/2018 (BOE 2018), tanto anual como quinquenalmente. En este proceso, los factores han sido calculados para las emisiones de los hornos de las cementeras españolas desde la perspectiva tecnológica y ambiental (Mora-Peris et al. en proceso) con una metodología característica de este sector del cemento y con base en datos reales. Los resultados obtenidos permiten disponer de un método de cálculo estándar para mejorar el conocimiento de las características, cantidades y frecuencias de las emisiones atmosféricas significativas del sector.

\section{MATERIALES Y MÉTODOS}

\section{Selección de contaminantes}

Los FE deben ser lo más representativos del sistema industrial en cuestión y basarse en datos reales de la actividad específica. Para el caso en estudio, emisiones de la industria cementera, existen numerosas referencias bibliográficas sobre factores de emisión que, además, son propuestos por organismos de prestigio internacional, entre los que cabe destacar la Guía de inventarios de emisiones de contaminantes atmosféricos EMEP/AEMA(EMEP/EEA 2013), una guía indispensable para la realización de inventarios nacionales de emisiones en Europa o el Documento de referencia sobre las mejores técnicas disponibles en la industria de fabricación de cemento, cal y óxido de magnesio (BREF), el cual describe los FE calculados a partir de datos de concentración (Schorcht et al. 2013).

Para este estudio se llevó a cabo una revisión del estado del arte disponible, prosiguiendo con la determinación de las sustancias contaminantes más significativas emitidas por las fábricas de cemento $\mathrm{y}$, por consiguiente, los FE correspondientes más representativos del sector, todo ello en función de los datos de actividad y la legislación vigente.

Recopilando la legislación aplicable al sector cementero en España relacionada con las emisiones (Canales et al. 2003) y con la información disponible en el Inventario Nacional y el Registro PRTR-España (Canales et al. 2003), se determinó que las sustancias más características seleccionadas y agrupadas por familias son las reflejadas en el cuadro I.

Con esta información de todos los contaminantes recogidos en el BREF (Canales et al. 2003) como referencia (si bien se consideran los datos emitidos por las instalaciones que generan dichos contaminantes, reflejados anteriormente), el artículo se enfoca a la selección de aquellos cuyos valores límite para la incineración de cemento están señalados en el RD 815/2013 (BOE 2013) (cuadro II).

Durante la revisión del estado del arte que se realizó en este estudio, se observaron todos los aspectos posibles en los que influyen los contaminantes característicos de la industria cementera. En el cuadro III se muestran los impactos causados por las sustancias emitidas que forman parte de este estudio. 
CUADRO I. AGRUPACIÓN CONCEPTUAL DE CONTAMINANTES POR FAMILIAS EN EL SECTOR CEMENTERO.

\begin{tabular}{|c|c|c|c|c|}
\hline $\begin{array}{c}\text { Familia de } \\
\text { contaminantes }\end{array}$ & $\begin{array}{l}\text { Compuestos } \\
\text { ácidos }\end{array}$ & $\begin{array}{c}\text { Metales pesados } \\
\text { y metales de } \\
\text { transición }\end{array}$ & $\begin{array}{c}\text { Compuestos } \\
\text { orgánicos }\end{array}$ & Sólidos \\
\hline Contaminantes & $\begin{array}{c}\mathrm{CO} \\
\mathrm{NO}_{\mathrm{x}} \\
\mathrm{SO}_{\mathrm{x}} \\
\mathrm{N}_{2} \mathrm{O} \\
\mathrm{NH}_{3} \\
\mathrm{CHl} \\
\mathrm{HF}\end{array}$ & $\begin{array}{c}\mathrm{As} \\
\mathrm{Cd} \\
\mathrm{Cr} \\
\mathrm{Cu} \\
\mathrm{Ni} \\
\mathrm{Pb} \\
\mathrm{Tl} \\
\mathrm{Sb} \\
\mathrm{Co} \\
\mathrm{V} \\
\mathrm{Mn} \\
\mathrm{Se} \\
\mathrm{Hg} \\
\mathrm{Zn}\end{array}$ & $\begin{array}{c}\text { NMVOC } \\
\text { Benceno } \\
\text { COT } \\
\mathrm{CH}_{4} \\
\mathrm{PAH} \\
\mathrm{PCB} \\
\mathrm{PCCD} \\
\mathrm{PCCF}\end{array}$ & $\begin{array}{c}\text { Partículas } \\
\text { totales } \\
\mathrm{PM}_{10}\end{array}$ \\
\hline
\end{tabular}

NMVOC: compuestos orgánicos volátiles distintos del metano, COT: carbono orgánico total, PAH: hidrocarburos aromáticos policíclicos, PCB: bifenilos policlorados, PCDD: policlorodibenzodioxinas, PCDF: policlorodibenzofuranos.

CUADRO II. CONTAMINANTES CON VALORES LÍMITE DE EMISIÓN SEGÚN EL RD 815/2013.

\begin{tabular}{lc}
\hline Contaminantes & Valores límite $\left(\mathrm{mg} / \mathrm{Nm}^{3}\right)$ \\
\hline Partículas totales & 30 \\
$\mathrm{HCl}$ & 10 \\
$\mathrm{HF}$ & 1 \\
$\mathrm{NOx}$ & 500 \\
$\mathrm{Cd}+\mathrm{Tl}$ & 0.05 \\
$\mathrm{Hg}$ & 0.05 \\
$\mathrm{Sb}+\mathrm{As}+\mathrm{Pb}+\mathrm{Cr}+\mathrm{Co}+\mathrm{Cu}+\mathrm{Mn}+\mathrm{Ni}+\mathrm{V}$ & 0.5 \\
& Valores límite $\left(\mathrm{ng} / \mathrm{Nm}^{3}\right)$ \\
Dioxinas y furanos & 0.1 \\
\hline
\end{tabular}

\section{Metodología de medición}

Como se ha tratado en el apartado anterior, existe abundante literatura sobre FE y metodologías de gran renombre para el cálculo de los mismos, por ejemplo el sistema de medición de la Asociación de Fabricantes de Cemento de Alemania (VDZ, por sus siglas en alemán), que consiste en una nube de puntos de cada contaminante en la que se muestra la concentración y se procede tanto en medición continua como discontinua (VDZ 2010). Sin embargo, como se ha destacado, estas técnicas de medición reconocidas no necesariamente son aplicables al sector cementero español, ya que tanto los datos como su uso y las condiciones de medición pueden ser distintas a las internacionales. Esto, porque la normalización puede diferir entre países (normas DIN, UNE, ASTM); un ejemplo de ello es la toma de muestras en continuo para mercurio o dioxinas y furanos en el sector de la incineración respecto al presente caso de estudio.

Una metodología de evaluación correcta de FE se basa en la caracterización completa de las emisiones y su medición con métodos normalizados, lo que requiere determinar la concentración de los contaminantes significativos previamente descritos. Hay que tener en consideración que existen factores como representatividad de la muestra, tiempo de muestreo, acondicionamiento de equipos, fases de contaminantes emitidos (sólida y gaseosa) y condiciones de referencia que influyen en la medición, tal y como está descrito en las mejores técnicas disponibles en España para la fabricación de cemento (Canales et al. 2003).

De acuerdo con lo establecido tanto en la Autorización Ambiental Integrada (AAI) como en las legislaciones autonómicas pertinentes y en la legislación vigente (Ley 34/2007, RD 100/2011 y RD 815/2013) (BOE 2007, 2011, 2013), los propietarios de las instalaciones deben realizar obligatoriamente controles internos y externos, y llevar un control exhaustivo de todos los datos obtenidos. Ambos tipos de medida tienen como objetivo la comprobación y verificación del funcionamiento de sistemas de corrección y prevención, seguimiento de la contaminación y valores límite de emisión, entre otros. 
CUADRO III. DETERMINACIÓN DE CADA IMPACTO PROVOCADO POR CADA CONTAMINANTE.

\begin{tabular}{|c|c|c|c|}
\hline Contaminante & Impacto & Proceso & Referencia \\
\hline Mercurio (Hg) & \multirow{5}{*}{$\begin{array}{l}\text { Salud } \\
\text { Bioacumulación } \\
\text { No degradable } \\
\text { Biomagnificación }\end{array}$} & \multirow{5}{*}{$\begin{array}{l}\text { Combustión combustible } \\
\text { (carbón y otros), } \\
\text { calcinación de mineral } \\
\text { y uso de energía }\end{array}$} & \multirow{5}{*}{$\begin{array}{c}\text { Cipurkovicet al. } 2014 \\
\text { Nirel y Pasquini } 2010 \\
\text { Al-Khashman y Shawabkeh } 2006 \\
\text { Karbowska } 2016\end{array}$} \\
\hline Plomo (Pb) & & & \\
\hline Cadmio (Cd) & & & \\
\hline Cobre $(\mathrm{Cu})$ & & & \\
\hline Cromo $(\mathrm{Cr})$ & & & \\
\hline $\mathrm{NOx}\left(\mathrm{NO}\right.$ y $\left.\mathrm{NO}_{2}\right)$ & $\begin{array}{l}\text { Acidificación de } \\
\text { ecosistemas } \\
\text { Eutrofización }\end{array}$ & $\begin{array}{l}\text { Combustión de combustible, } \\
\text { calcinación de mineral y } \\
\text { uso de energía }\end{array}$ & $\begin{array}{l}\text { Drossman } 2002 \\
\text { Lei et al. } 2011 \\
\text { UNFCCC } 2016 \\
\text { Roe et al. } 2004\end{array}$ \\
\hline $\begin{array}{l}\text { Dioxinas y } \\
\text { furanos }\end{array}$ & $\begin{array}{c}\text { Compuestos } \\
\text { orgánicos persistentes } \\
(\mathrm{COP})\end{array}$ & $\begin{array}{c}\text { Combustión de combustible } \\
\text { y residuo }\end{array}$ & $\begin{array}{c}\text { Conesa et al. } 2016 \\
\text { Sánchez-Palencia et al. } 2015 \\
\text { Ruiz. et al. } 2005\end{array}$ \\
\hline PST & $\begin{array}{l}\text { Partículas en } \\
\text { suspensión }\end{array}$ & Uso de energía, minería & $\begin{array}{l}\text { Drossman } 2002 \\
\text { Lei et al. } 2011 \\
\text { UNFCCC } 2016 \\
\text { Chang-Tang } 2004\end{array}$ \\
\hline
\end{tabular}

PST: partículas suspendidas totales.

En los controles internos, las medidas de emisiones, realizadas por el titular de la industria, deben proceder de acuerdo con lo establecido en la administración competente; es decir, según lo plasmado en la legislación vigente de la comunidad autónoma donde está ubicada la planta. En cuanto a los controles externos, las mediciones deben ser realizadas por los organismos que establezca la comunidad autónoma, los cuales son entidades totalmente capacitadas técnicamente y acreditas por la Entidad Nacional de Acreditación (ENAC), según lo establecido en la norma UNE-EN ISO/IEC 17020:2012 (UNE 2012).

Para ambos tipos de control, el criterio de medición y de elaboración del informe de control se realiza de acuerdo con lo estipulado en la norma UNE-EN 15259:2008 (UNE 2008). Asimismo, el muestreo, análisis de contaminantes y métodos de medición se realizan conforme a las normas existentes del Comités Europeo de Normalización (CEN).

\section{Mediciones en continuo y puntuales}

Los sistemas de medición y control de emisiones son de dos tipos: medición en discontinuo y medición en continuo, mediante sistemas continuos de medida, también conocidos como sistemas automáticos de medida en continuo (SAM). En el sector cementero español, las Autorizaciones Ambientales Integradas obligan a las instalaciones a contar con un SAM.
El SAM es un equipo que mide en continuo los parámetros físicos y químicos relevantes para obtener una cuantificación correcta de la emisión de un contaminante. La medición en continuo, según lo estipulado, mide partículas suspendidas totales (PST), $\mathrm{HCl}, \mathrm{HF}, \mathrm{NO}_{\mathrm{x}}, \mathrm{SO}_{2}$ y carbono orgánico total (COT) en hornos en los que se coincineran los residuos (BOE 2013), y se recomienda usarla para medir CO.

La medición discontinua consiste en obtener, mediante técnicas y equipos de filtración, extracción, absorción y adsorción, una cantidad de muestra, siempre representativa, de la corriente emisora. De esta manera se obtienen mediciones que permiten la determinación puntual de parámetros específicos en un determinado tiempo limitado.

En el presente estudio, todos los valores límite a los que hace referencia el cuadro II se establecieron como valores medios durante un periodo de tiempo. En el caso de PST, $\mathrm{HCl}, \mathrm{HF}$ y $\mathrm{NO}_{\mathrm{x}}$, se consideró una medida continua; para metales pesados $(\mathrm{Cd}+\mathrm{Tl}, \mathrm{Hg}$, $\mathrm{Sb}+\mathrm{As}+\mathrm{Pb}+\mathrm{Cr}+\mathrm{Co}+\mathrm{Cu}+\mathrm{Mn}+\mathrm{Ni}+\mathrm{V})$ se determinó un rango de tiempo variable (30 min- $8 \mathrm{~h}$ ). Para dioxinas y furanos (PCCD y PCCF), el intervalo de tiempo varió de 6 a 8 h, siendo estos valores el mínimo y el máximo.

Queda claro que el número de mediciones requeridas para obtener resultados totalmente 
comparables, significativos y representativos dependerá de cada caso y cada contaminante, y por ende de las condiciones en las que se realizan las mediciones.

\section{Metodología plurianual seguida en el presente estudio}

La metodología empleada parte de una revisión extensa de la literatura existente sobre metodología de cálculo y evaluación de FE. Tras este paso se seleccionaron los contaminantes más característicos de la industria cementera y se procedió a pedir los datos de medición de dichos contaminantes a todas las instalaciones del sector en España. Estos números se agruparon por sustancias y por años y quinquenios a partir de 2007 de la siguiente manera: 2007-2011, 2008-2012, 2009-2013, 2010-2014, 2011-2015, 2012-2016, 2013-2017 y 2014-2018.

Una vez recibidos los datos y agrupados de la manera descrita, se continuó con el tratamiento de la información por medio de una herramienta estadística y se calcularon y evaluaron los FE de cada sustancia contaminante de todas las fábricas con las ecuaciones pertinentes.

Por otra parte, la metodología de toma de muestras a lo largo de los años se ha ido modificando e incorporando la legislación vigente de ese periodo.

Tanto el muestreo y el análisis de las sustancias como la verificación de la calidad de los sistemas de medición automática y los métodos de medición de referencia para su calibración se llevaron a cabo de acuerdo con las normas CEN. En ausencia de éstas, se aplicaron las normas ISO o, en su defecto, normas nacionales o internacionales, con el fin de garantizar la obtención de datos de suficiente calidad científica.

Este aseguramiento de la calidad de los sistemas de medición ha sido uno de los grandes objetivos del presente trabajo, ya que la aplicación de métodos normalizados de medición para algunos componentes, aplicada a mediciones estacionales, han generado fallos e incertidumbres en el desarrollo de la investigación.

La medición es anual; sin embargo, los resultados medidos se agrupan por quinquenios y se obtienen las medias. De esta manera se evita la afluencia de valores estadísticamente anormales, motivados por la existencia de errores en la selección del método de medición o en la toma de muestras.

\section{Cálculo de los factores de emisión}

Para caracterizar las emisiones hay que calcular la concentración de contaminantes, obteniendo los datos de una serie de parámetros característicos de la corriente emisora y otros necesarios para el flujo volumétrico.

El cálculo de la carga contaminante anual de emisión se realiza a partir de los factores de emisión del propio sector industrial y de datos de la actividad como balances de materia, de manera que se determina con la ecuación 1 :

$$
\begin{aligned}
& \text { Carga contaminante } \\
& \text { anual emitida }
\end{aligned}\left(\frac{\mathrm{kg}}{\text { año }}\right)-F E_{\text {contaminante }} * D_{\text {actividad }}
$$

donde $F E_{\text {contaminante }}$ es el factor de emisión para el contaminante $i$ y $D_{\text {actividad }}$ es el dato de la actividad. El cálculo de los FE por medio de la herramienta estadística diseñada, la cual se mencionó anteriormente, se lleva a cabo mediante la aplicación secuencial de las ecuaciones 2, 3, 4 y 5 .

Cálculo de la carga contaminante específica por horno, contaminante y año:

$F E_{\text {horno i,añox }}=\frac{C i_{x} * Q i_{x} * 10^{-6}}{P k_{\text {horno i, iñox }}}$

donde $F E_{\text {horno i,año x }}$ es la carga contaminante específica o factor de emisión del horno $i$ para el año $x(\mathrm{~kg}$ de contaminante/t de clínker); $C i_{\mathrm{x}}$ es la concentración media del contaminante en el horno $i$ en el año $x\left(\mathrm{mg} / \mathrm{Nm}^{3}\right)$, calculada a partir de la concentración del contaminante corregida al $10 \%$ de $\mathrm{O}_{2}$ y en base seca; $Q i_{\mathrm{x}}$ es el caudal anual del horno $i$ en el año $x$ $\left(\mathrm{Nm}^{3} / \mathrm{año}\right)$ corregido al $10 \%$ de $\mathrm{O}_{2}$ y en base seca;

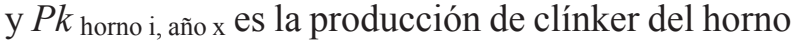
$i$ en el año $x$ (t clínker/año).

Cálculo de la carga contaminante específica por fábrica, contaminante y año:

$F E_{\text {fäbrica f,año } x}=\sum_{1}^{n} \frac{(\text { FEhorno i, año } x * \text { pk horno i, año } x)}{\left.\sum \text { (Pk horno i,año } x\right)}$

donde $F E$ fabrica f, año x es la carga contaminante específica o factor de emisión de la fábrica $f$ en el año $x$ (kg de contaminante/t clínker); $F E$ horno i, año x es la carga contaminante específica del horno $i$ para el año $x$ ( $\mathrm{kg}$ de contaminante/t clínker); $P k$ horno i, año $\mathrm{x}$ es la producción de clínker del horno $i$ en el año $x(\mathrm{t}$ clínker/año); y $n$ es el número de hornos existentes en la fábrica $f$ en el año $x$.

Cálculo de la carga contaminante específica por media del sector por contaminante y año:

$F E$ sector $_{\text {año } x}=\sum_{1}^{f} \frac{F E \text { fäbrica f, año } x * P K \text { fábrica f, año } x}{\sum(\text { Pkfäbrica f,año } x)}$

Cálculo de la carga contaminante específica media quinquenal del sector por contaminante (al año $x$ se le asigna la correspondiente al periodo $x-4 \mathrm{a} x$ ): 


$$
\text { FEQ } \text { sector }_{\text {año } x}=\sum_{1}^{x} \frac{\text { FEsector, año } x * P k \text { sector, año } x}{\sum(\text { Pk sector,año } x)}
$$

donde FEQ sector año $x$ es la carga contaminante específica o factor de emisión quinquenal (media móvil) del sector en el año $x$ (kg de contaminante $/ \mathrm{t}$ clínker); FE sector año $x$ es la carga contaminante específica o factor de emisión del sector en el año $x$ ( $\mathrm{kg}$ de contaminante/t clínker), y $P k$ sector, año $x$ es la producción de clínker del sector en el año $x$ ( $\mathrm{t}$ clínker/año).

La información remitida por los titulares de las cementeras está compuesta por concentración anual del contaminante para cada horno en $\mathrm{mg} / \mathrm{Nm}^{3}$, calculada a partir de la concentración del contaminante en cuestión, en base seca, en condiciones normales y al $10 \%$ de $\mathrm{O}_{2}$. Respecto de los contaminantes medidos en continuo, la concentración media anual se ha obtenido de los datos proporcionados por el SAM, mientras que para los restantes se han utilizado mediciones puntuales.

El cálculo estadístico que forma parte de esta metodología se compone de una media móvil quinquenal sectorial, medias ponderadas con base en la producción de clínker $y$, por último, el tamaño de muestra de cada contaminante, que siempre debe ser representativa para que los resultados sean válidos.

En líneas generales, todo método de medición tiene un límite de cara a la concentración más baja que esté capacitado para medir. Por ello es frecuente diferenciar entre límite de detección (LOD) y límite de cuantificación (LOQ), de manera que se utiliza un método de medida que tenga un límite inferior al $10 \%$ del valor límite de emisión implantado para el proceso. Para el caso en que existan valores por debajo del límite de detección se proponen las siguientes opciones:

Usar en los cálculos el valor límite de detección, lo que tiende a sobreestimar los resultados.

Usar en los cálculos la mitad del límite de detección u otro valor, tendiendo tanto a sobreestimar como subestimar los resultados.

Usar en los cálculos el valor cero, tendiendo a subestimar los resultados.

En caso de que no se quiera usar alguna de estas opciones, se puede proceder al cálculo mediante la ecuación 6:

Valor a utilizar $=(100 \%-A) \times L D$

donde $A$ es el porcentaje de las muestras medidas con resultados inferiores al LOD del parámetro medido. Con la ecuación 6 se determinan los FE.

Puede darse el caso de que para un mismo contaminante existan varias mediciones $\mathrm{y}$, además, los valores de algunas de éstas estén por debajo del límite de detección. En este caso el valor a utilizar para cada una de las muestras se determinará de acuerdo con los siguientes casos:

- El valor que proporciona el laboratorio está por debajo del límite de detección. En estos casos se utiliza la ecuación 7 para cada muestra:

$$
\text { Valor a utilizar }=(100 \%-A) \times L D^{\prime}
$$

- El valor que proporciona el laboratorio está por encima del límite de detección. En este caso se utiliza dicho valor.

El valor medio asignado al contaminante:

- Cero si el valor medio de los valores obtenidos en cada muestra es inferior al menor límite de detección proporcionado por el laboratorio.

- El valor medio de los valores obtenidos, en caso de que este valor sea superior o igual al menor límite de detección que proporciona el laboratorio.

El límite de detección menor es aquel que tenga el valor más bajo entre los proporcionados por el laboratorio para todas las muestras.

\section{RESULTADOS Y DISCUSIÓN}

Para la interpretación de los datos obtenidos a partir de la Ecuación 5 (Cuadro IV) se debe tener en cuenta que el método de toma de muestras evolucionó a lo largo del intervalo de tiempo establecido en la investigación (2007-2018). Uno de los factores más importantes ha sido la evolución de los métodos normalizados de medición adecuados para determinados contaminantes en fuentes estacionarias. El uso de un método inadecuado puede generar errores en la medición superiores al $100 \%$ del valor medido. Por ello la homogenización normalizada de las mediciones ha sido uno de los principales avances del proyecto. También las incertidumbres y los errores de estimación en los cálculos anuales han evolucionado, de tal manera que los errores han ido disminuyendo. De la misma manera, con la evolución de la tecnología se han incorporado nuevas técnicas de mejora en las instalaciones, por lo cual debe destacarse que posiblemente alguna medida expuesta resalte por estos motivos.

Por otro lado, en el caso de algunos contaminantes seleccionados (por ejemplo, el $\mathrm{N}_{2} \mathrm{O}$ ) no hay personas acreditadas por la ENAC para la realización de ensayos en España, mientras que para otros contaminantes las mediciones no siguen las normas CEN sino que se 
CUADRO IV. FE POR kg/t DE CLÍNKER EN FÁBRICAS DE VÍA SECA, CON MTD 2019

\begin{tabular}{|c|c|c|}
\hline Contaminantes & & FE ( $\mathrm{kg} / \mathrm{t}$ clínker) \\
\hline \multirow{7}{*}{$\begin{array}{l}\text { Compuestos } \\
\text { ácidos }\end{array}$} & $\mathrm{CO}$ & 2.2878978 \\
\hline & $\mathrm{NO}_{\mathrm{x}}$ & 1.4717519 \\
\hline & $\mathrm{SO}_{\mathrm{x}}$ & 0.1329718 \\
\hline & $\mathrm{N}_{2} \mathrm{O}$ & 0.0041555 \\
\hline & $\mathrm{NH}_{3}$ & 0.0378856 \\
\hline & $\mathrm{HCl}$ & 0.0038552 \\
\hline & $\mathrm{HF}$ & 0.0002619 \\
\hline \multirow{14}{*}{$\begin{array}{l}\text { Metales pesados } \\
\text { y metales } \\
\text { de transición }\end{array}$} & As & 0.0000035 \\
\hline & $\mathrm{Cd}$ & 0.0000028 \\
\hline & $\mathrm{Cr}$ & 0.0000304 \\
\hline & $\mathrm{Cu}$ & 0.0000255 \\
\hline & $\mathrm{Ni}$ & 0.0000219 \\
\hline & $\mathrm{Pb}$ & 0.0000331 \\
\hline & $\mathrm{Tl}$ & 0.0000122 \\
\hline & $\mathrm{Sb}$ & 0.0000051 \\
\hline & $\mathrm{Co}$ & 0.0000042 \\
\hline & $\mathrm{V}$ & 0.0000044 \\
\hline & $\mathrm{Mn}$ & 0.0000261 \\
\hline & $\mathrm{Se}$ & 0.0000217 \\
\hline & $\mathrm{Hg}$ & 0.0000235 \\
\hline & $\mathrm{Zn}$ & 0.0000862 \\
\hline \multirow{7}{*}{$\begin{array}{l}\text { Compuestos } \\
\text { orgánicos }\end{array}$} & NMVOC & 0.015911 \\
\hline & Benceno & 0.0017948 \\
\hline & COT & 0.0455842 \\
\hline & $\mathrm{CH}_{4}$ & 0.0012577 \\
\hline & PAH & 0.0000122 \\
\hline & PCB & 0.0000000003 \\
\hline & PCDD/F (ng/t clínker) & 27.7519245 \\
\hline \multirow[t]{2}{*}{ Sólidos } & Partículas totales & 0.020267 \\
\hline & $\mathrm{PM}_{10}$ & 0.0080107 \\
\hline
\end{tabular}

NMVOC: compuestos orgánicos volátiles distintos del metano, COT: carbono orgánico total, PAH: hidrocarburos aromáticos policíclicos, PCB: bifenilos policlorados, PCDD/F: policlorodibenzodioxinas/ policlorodibenzofuranos.

han tenido que adaptar de acuerdo con las normas ISO nacionales o internacionales, como EPA, ASTM, etc. Por tanto las variaciones de índices son incongruentes o pueden inducir errores de interpretación.

Para la interpretación de datos se toma como base 100 la media del quinquenio 2007-2011. En el análisis global de resultados se aprecia una gran disminución de los factores emitidos por todos los componentes.

Centrando el análisis en los valores obtenidos para compuestos ácidos $\left(\mathrm{NO}_{\mathrm{x}}\right.$ como $\left.\mathrm{SO}_{2}\right)$ (Fig. 1), hay una disminución del índice con tendencia a ser constante, debido a la implementación de MTD, tales como sistemas de desulfuración y reducción no catalítica de dióxido de nitrógeno. Lo mismo ocurre con el $\mathrm{HCl}$ y el $\mathrm{HF}$, cuyas emisiones disminuyen y sus medidas se basan en sistemas de control de $\mathrm{Cl}$ y $\mathrm{F}$ en materias primas y combustibles.

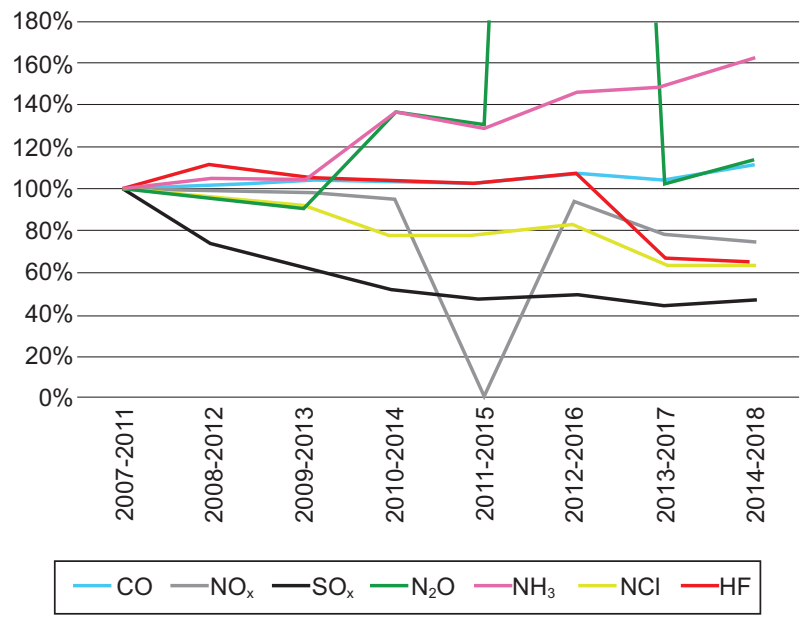

Fig. 1. Evolución de emisiones de compuestos ácidos respecto al año quinquenio base de los factores.

A pesar de todo, existe un incremento de las emisiones de $\mathrm{CO}$ y $\mathrm{NH}_{3}$ debido al ajuste de los sistemas de combustión y a emisiones difusas de amoniaco generadas por la imposición de sistemas no catalíticos de reducción de $\mathrm{NO}_{\mathrm{x}}$ en los que se inyecta amonio. El $\mathrm{N}_{2} \mathrm{O}$ presenta un comportamiento errático en la medición, con valores en aumento, debido principalmente a errores producidos en los métodos de medición no normalizados, lo que determina una anomalía estadística a la hora de su medición.

Para los componentes pertenecientes a la familia de los metales pesados y de transición (Fig. 2) se observa en general una amplia variabilidad, pero se aprecia una cierta constancia para cada contaminante específico; a causa de ello (como se explicó antes), la calidad de las mediciones efectuadas en la primera campaña, cuyos valores se toman como referencia y a partir las cuales se efectúan los cálculos de índices, era inadecuada, ya que era la dictada por la legislación vigente del momento. Esto provoca una dispersión estadística en las medidas puntuales, puesto que los métodos de medición se tienen que adaptar en función de las modificaciones de las normativas vigentes.

En el caso de los compuestos orgánicos (Fig. 3), éstos presentan una reducción global significativa, aunque en algunos casos, tales como $\mathrm{PCDD} / \mathrm{F}$ y bencenos, las incertidumbres de métodos de medición y las interrelaciones entre compuestos pueden generar incrementos en las incertidumbres en los valores. 


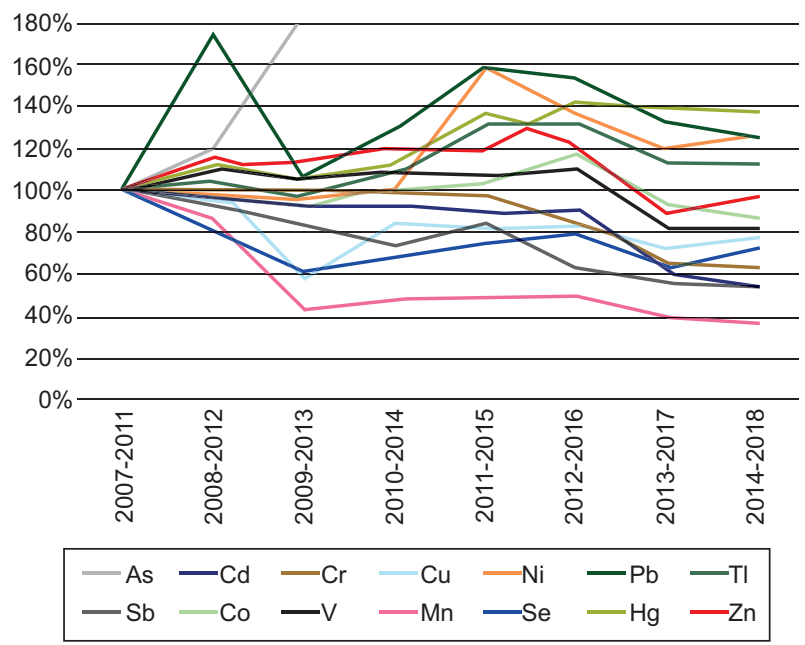

Fig. 2. Evolución de emisiones de metales pesados y metales de transición respecto al año quinquenio base de los factores.

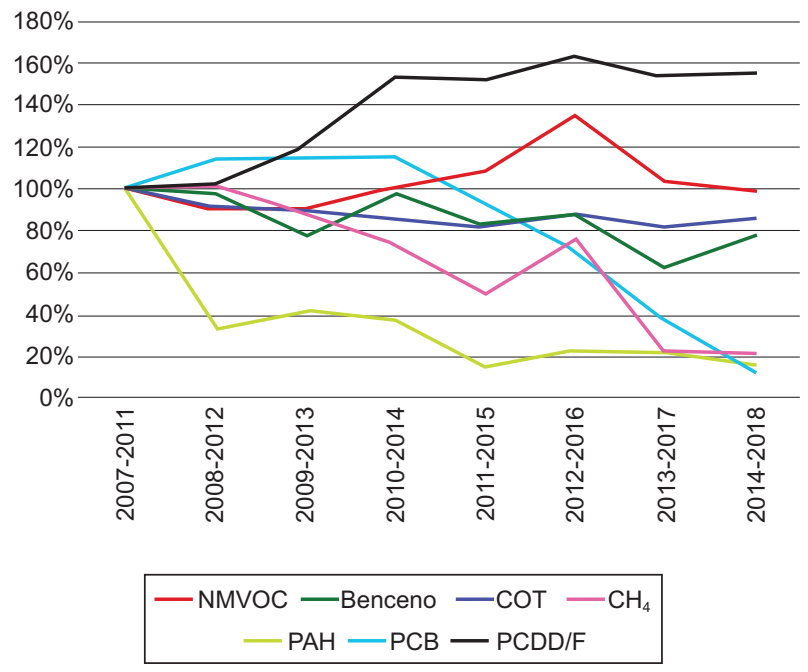

Fig. 3. Evolución de emisiones de compuestos orgánicos respecto al año quinquenio base de los factores.

En cuanto a las partículas sólidas (Fig. 4), se aprecia un característico decremento de emisiones, fomentado primordialmente por el establecimiento de equipos como filtros de mangas de última generación (los cuales se engloban como MTD) debido al avance tecnológico de los últimos años.

Los factores de emisión por producción de cemento en fábricas de vía seca, que incorporan las MTD del último año, son los mostrados en el cuadro I. Esos resultados recogen la media de valores de los últimos cinco años.

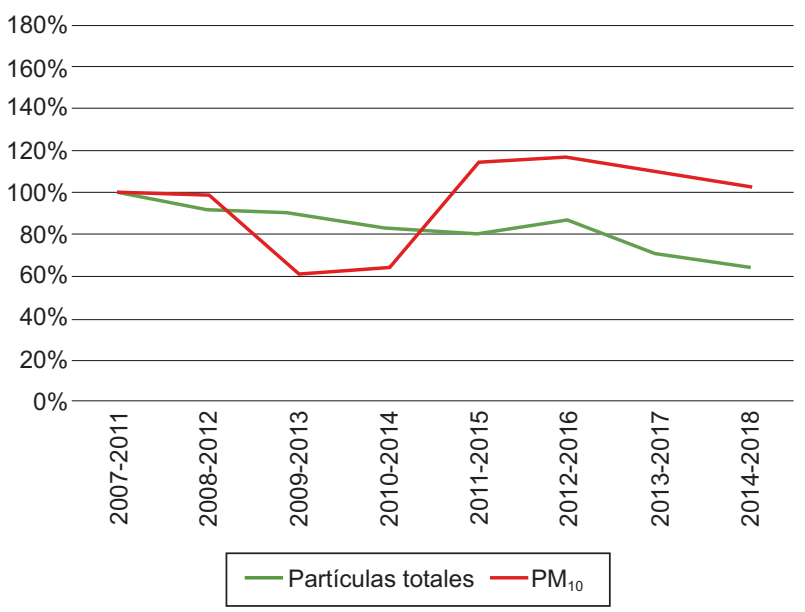

Fig. 4. Evolución de emisiones de partículas sólidas respecto al año quinquenio base de los factores

\section{CONCLUSIONES}

La industria del cemento detectó que los FE expuestos en fuentes bibliográficas, los cuales se han utilizado de manera habitual tanto en inventarios nacionales y europeos como en protocolos internacionales, no eran equiparables a las técnicas implantadas en las fábricas ni se adaptaban a las normativas vigentes. Por ende, se vio la necesidad de desarrollar una metodología, adecuada a las condiciones del sector cementero español, que definiera los FE propios, de tal manera que permitiera obtener cálculos más característicos y ajustados a las emisiones reales y que hicieran más comparables los resultados entre sí.

Se ha determinado el cálculo de FE en instalaciones cementeras de hornos en vía seca y que siguen directrices de las MTD. Por el contrario, no se consideran aquellas industrias de las que toman datos EMEP/CORINAIR; fábricas de hornos tanto en vía seca como húmeda, y que además no desarrollan su actividad con equipos que emplean criterios establecidos en las MTD.

Para ello, y tras seleccionar los contaminantes más característicos emitidos, se diseñó la metodología para obtener los FE, teniendo en cuenta la realidad de la industria cementera española desde el punto de vista tecnológico y ambiental. De esta manera se incorporó toda la información existente disponible sobre emisiones y la evolución continua y real de la implantación de las MTD del sector en estudio.

Los FE obtenidos en este trabajo se ajustan a las características del sector cementero español en mayor medida que los propios FE teóricos procedentes de las distintas fuentes bibliográfica. Esto se debe a que los 
primeros se basan en datos derivados de la operación real de las cementeras españolas, los cuales no sólo son reales sino que, además, pertenecen a un rango de años largo y reciente.

Este estudio permite, adicionalmente, que los datos proporcionados sobre cargas de contaminantes anuales entre las distintas fábricas sean comparables y coherentes con los diversos instrumentos y requisitos legales de información ambientales, tales como inventarios nacionales, registros PRTR y otros.

Con los resultados obtenidos se puede afirmar que la industria cementera tiene a su disposición, para la evaluación de FE, una herramienta consistente y representativa debido a su metodología y por la muestra temporal y de las instalaciones, respectivamente. Cuenta además con un instrumento para la armonización de criterios de metodología de medición y FE atmosféricos sectoriales. Esta metodología es aplicable a conjuntos de fábricas de cemento en cualquier país que considere necesario disponer de factores reales actualizados para las tecnologías existentes.

A pesar de la existencia de esta nueva metodología, se recomienda la revisión periódica, para tener en cuenta los posibles cambios que puedan ocurrir en el sector cementero durante los próximos años, sobre todo en áreas técnicas y ambientales, referentes a instalaciones, requisitos y prácticas de seguimiento.

\section{REFERENCIAS}

Abril G.A., Wannaz E.D., Mateos A.C. y Pignata M.L. (2014). Biomonitoring of airborne particulate matter emitted from a cement plant and comparison with dispersion modelling results. Atmos. Environ. 82, 154163. https://doi.org/10.1016/j.atmosenv.2013.10.020

Ali M.B., Saidur R. y Hossain M.S. (2011). A review on emission analysis in cement industries. Renew. Sust. Energ. Rev. 15 (5), 2252-2261. https://doi. org/10.1016/j.rser.2011.02.014

Arden Pope C., Thun M.J., Namboodiri M.M., Dockery D.W., Evans J.S., Speizer F.E. y Heath C.W. (1995). Particulate air pollution as a predictor of mortality in a prospective study of U.S. adults. Am. J. Respir. Crit. Care Med. 151, 669-674. https://doi.org/10.1164/ ajrccm/151.3_Pt_1.669

Al-Khashman O. y Shawabkeh R. (2006). Metals distribution in soils around the cement factory in southern Jordan. Environ. Pollut. 140 (3), 389-394. https://doi. org/10.1016/j.envpol.2005.08.023

Barba A., Gazulla M.F., Gómez M.P., Puertas F., GarcíaDíaz I., Palacios M. y Martínez-Ramírez S. (2006).
Residuos cerámicos para su posible uso como materia prima en la fabricación de clínker de cemento Portland: caracterización y activación alcalina. Materiales de Construcción 56 (281), 73-84 https://doi.org/10.3989/ mc.2006.v56.i281.94

Barba A., Gazulla M.F., Gómez M.P., Puertas F., GarcíaDíaz I., Palacios M. y Martínez-Ramírez S. (2007). Empleo de residuos cerámicos como materia prima alternativa para la fabricación de clínker de cemento Portland. Cemento Hormigón 907, 20-34.

Bertoldi M., Borgini A., Tittarelli A., Fattore E., Cau A., Fanelli R. y Crosignani P. (2012). Health effects for the population living near a cement plant: An epidemiological assessment. Environ. Int. 41, 1-7. https:// doi.org/10.1016/j.envint.2011.12.005

BOE (2007). Ley 34/2007, de 15 de noviembre, de calidad del aire y protección de la atmósfera. Jefatura del Estado. Boletín Oficial del Estado, 17 de noviembre.

BOE (2011). Real Decreto 100/2011, de 28 de enero, por el que se actualiza el catálogo de actividades potencialmente contaminadoras de la atmósfera y se establecen las disposiciones básicas para su aplicación. Ministerio de Medio Ambiente, y Medio Rural y Marino. Boletín Oficial del Estado, 29 de enero1.

BOE (2013). Real Decreto 815/2013, de 18 de octubre, por el que se aprueba el Reglamento de emisiones industriales y de desarrollo de la Ley 16/2002, de 1 de julio, de prevención y control integrados de la contaminación. Ministerio de Agricultura, Alimentación y Medio Ambiente. Boletín Oficial del Estado, 19 de octubre.

BOE (2018). Real Decreto 818/2018, de 6 de julio, sobre medidas para la reducción de las emisiones nacionales de determinados contaminantes atmosféricos. Ministerio para la Transición Ecológica. Boletín Oficial del Estado, 7 de julio.

Canales C., Giménez O., Avellaneda A., Monfá E., Silva S., Mora P., Romay M. y Serra M.A. (2003). Guía de mejores técnicas disponibles en España de fabricación de cemento. 84-8320-264-6. Ministerio de Medio Ambiente, Madrid, España.

Canpolat B., Atimtay A., Munlafalioglu I., Kalafatoglu E. y Ekinci E. (2002). Emission factors of cement industry in Turkey. Water Air Soil Pollut. 138, 235-252. https:// doi.org/10.1023/A:1015502131180

Carrasco F., Bredin N. y Heitz M. (2010). Gaseous contaminant emissions as affected by burning scrap tires in cement manufacturing. J. Environ. Qual. 31 (5), 1484. https://doi.org/10.2134/jeq2002.1484

Chang-Tang C. (2004). Assesment of influential range and characteristics of fugitive dust in limestone extraction processes. J. Air Waste Manage. 54 (2), 141-149. https://doi.org/10.1080/10473289.2004.10470889 
Cipurkovic A., Trumic I., Hodzic Z., Selimbasic V. y Djozic A. (2014). Distribution of heavy metal in Portland cement production process. Adv. Appl. Sci. Res. 5 (6), 252-259.

Conesa J., Ortuño N., Abad E. y Rivera-Austrui J. (2016). Emissions of PCDD/Fs, PBDD/Fs, dioxin like PCBs and $\mathrm{PAH}$ from cement plant using a long-term monitoring system. Sci. Total Environ. 571, 435-443. https:// doi.org/10.1016/j.scitotenv.2016.07.009

DOUE (2010). Directiva 2010/75/UE del Parlamento Europeo y del Consejo, de 24 de noviembre de 2010, sobre las emisiones industriales (prevención y control integrados de la contaminación). Diario Oficial de la Unión Europea, 17 de diciembre.

DOUE (2013). Directiva 2013/163/UE: Decisión de ejecución de la comisión, de 26 de marzo de 2013, por la que se establecen las conclusiones sobre las mejores técnicas disponibles (MTD) para la fabricación de cemento, cal y óxido de magnesio conforme a la Directiva 2010/75/UE del Parlamento Europeo y del Consejo, sobre las emisiones industriales. Diario Oficial de la Unión Europea, 9 de abril.

Drossman H. (2002). Air pollution from energy use. Colorado College, Colorado Springs, EUA.

Estatista (2019). Consumo aparente de cemento en España de 2009 a 2018 (en miles de toneladas) [en línea]. https://es.statista.com/estadisticas/541563/materialesde-construccion-consumo-aparente-de-cemento-enespana/ 10/03/2020

European Environment Agency (2013). EMEP/EEA air pollutant emission inventory guidebook 2013. Luxemburgo.

García-Gusano D., Garraín D., Herrera I., Cabal H. y Lechón Y. (2015). Life cycle assessment of applying $\mathrm{CO}_{2}$ post-combustion capture to the Spanish cement production. J. Clean. Prod. 104 (1), 328-338. https:// doi.org/10.1016/j.jclepro.2013.11.056

Gupta R.K., Majumdar D., Trivedi J.V. y Bhanarkar A.D. (2012). Particulate matter and elemental emissions from a cement kiln. Fuel Process. Technol. 104, 343351. https://doi.org/10.1016/j.fuproc.2012.06.007

HEI (2000). Reanalysis of the Harvard Six Cities Study and the American Cancer Society Study of Particulate Air Pollution and Mortality. Special Report. Health Effects Institute, Boston, MA, EUA.

HEI (2002). Understanding the health effects of components of the particulate matter mix: Progress and next steps. HEI Perspectives. Health Effects Institute, Boston, MA, EUA.

Hoyos-Barreto A.E., Jiménez-Correa M.M., Ortiz-Muñoz A. y Montes de Correa C. (2008). Cement plant gaseous pollutant emission reduction technologies. Ing. Invest. 28 (3), 41-46. https://doi.org/10.15446/ing.investig
INECC (2013). Guía metodológica para la estimación de emisiones de fuentes fijas. Guía. Secretaría de Medio Ambiente y Recursos Naturales, Instituto Nacional de Ecología y Cambio Climático, México.

Karbowska B. (2016). Presence of thallium in the environment: sources of contaminations, distribution and monitoring methods. Environ. Monit. Assess. 188 (11), 640. https://doi.org/10.1007/s10661-016-5647-y

Karstensen K.H. (2008). Formation, release and control of dioxins in cement kilns. Chemosphere 70 (2008), 543-560. https://doi.org/10.1016/j.chemosphere.2007.06.081

Kong S., Jia Y., Lua B., Chen L., Han B., Li Z. y Bai Z. (2011). Characterization of $\mathrm{PM}_{10}$ source profiles for fugitive dust in Fushun, a city famous for coal. Atmos. Environ. 45 (30), 5351-65. https://doi.org/10.1016/j. atmosenv.2011.06.050

Laribi S., Dubois L., de Weireld G. y Thomas D. (2017). Optimization of the sour compression unit (SCU) process for $\mathrm{CO}_{2}$ purification applied to flue gases coming from oxy-combustion cement industries. Energy Procedia 114, 458-470. https://doi.org/10.1016/j. egypro.2017.03.1188

Lei Y., Zhang Q., Nielsen C. y He K. (2011). An inventory of primary air pollutants and $\mathrm{CO}_{2}$ emissions from cement production in China, 1990-2020. Atmos. Environ. 45 (1), 147-154. https://doi.org/10.1016/j. atmosenv.2010.09.034

Madlool N.A., Saidur R., Hossain M.S. y Rahim N.A. (2011). A critical review on energy use and savings in the cement industries. Renew. Sust. Energ. Rev. 15 (4), 2042-2060. https://doi.org/10.1016/j.rser.2011.01.005

McLellan B.C., Corder G.D., Giurco D.P. e Ishihara K.N. (2012). Renewable energy in the minerals industry: A review of global potential. J. Clean. Prod. 32, 32-44. https://doi.org/10.1016/j.jclepro.2012.03.016

Mora-Peris P., Romay M., Silva M. y Herrero C. (2008). El ciclo de la vida del cemento. Un puente a la sostenibilidad en la construcción. Cemento Hormigón 915, 66-75.

Mora-Peris P., Silva-Segovia S. y Tomé-Gil E. (2018). Establecimiento de una metodología para el cálculo de los factores de emisión atmosférica en el sector cementero. Memorias. XIV Congreso Internacional en Energía y Recursos Minerales, Sevilla.

Mora-Peris P., de Vicente-Mingarro I., Villa-Jiménez V. y Silva-Segovia S. (en proceso). Air emissions: A methodology for determining emission factors characteristics of the Spanish cement industry.

Nirel P. y Pasquini F. (2010). Differentiation of copper pollution origin: agricultural and urban sources. Department of Water Ecology, Ginebra, Suiza.

Pérez-Vidal H., Lunagómez-Rocha M.A. y Acosta-Pérez L.I. (2010). Analysis of total suspended particles (TSP) 
and breathable fraction-particulate matter $\left(\mathrm{PM}_{10}\right)$ in Cunduacán, Tabasco. Universidad y Ciencia 26 (2), 151-162.

Pey J., Pérez N., Querol X., Alastuey A., Cusack M. y Reche C. (2010). Intense winter atmospheric pollution episodes affecting the Western Mediterranean. Sci. Total Environ. 408 (8), 1951-1959. https://doi. org/10.1016/j.scitotenv.2010.01.052

Raffetti E., Treccani M. y Donato F. (2019). Cement plant emissions and health effects in the general population: a systematic review. Chemosphere 218, 211-222. https://doi.org/10.1016/j.chemosphere.2018.11.088

Richards G. y Agranovski I.E. (2017). Dioxin-like PCB emissions from cement kilns during the use of alternative fuels. J. Hazard. Mater. 323, 698-709. https://doi. org/10.1016/j.jhazmat.2016.10.040

Roe S., Spivey M., Lindquist H., Thesing K. y Strait R. (2004). Estimating ammonia emissions from anthropogenic nonagricultural sources. Emission Inventory Improvement Program, US Enviromental Protecion Agency, EUA.

Rovira J., Sierra J., Nadal M., Schuhmacher M. y Domingo J.L. (2014). Environmental levels of PCDD/ Fs and metals around a cement plant in Catalonia, Spain, before and after alternative fuel implementation. Assessment of human health risks. Sci. Total Environ. 485-486, 121-129. https://doi.org/10.1016/j. scitotenv.2014.03.061

Rovira J., Sierra J., Nadal M., Schuhmacher M. y Domingo J.L. (2018). Main components of $\mathrm{PM}_{10}$ in an area influenced by a cement plant in Catalonia, Spain: Seasonal and daily variations. Environ. Res. 165, 201-209. https://doi.org/10.1016/j.envres.2018.04.010

Ruiz L., Martínez M.Á. y de la Torre A. (2005). Estudio $\mathrm{y}$ resultados de la participación del sector cementero español en el Inventario Nacional de Dioxinas y Furanos (2000-2003). CIEMAT, Madrid, España, 199 pp.

Sana-Mehraj S., Bhat G.A. y Mehraj-Balkhi H. (2013). Cement factories and human health. Int. J. Curr. Res. Rev. 5(18), 47.

Sánchez-Palencia Y., Ortiz J., de Torres T. y Carro C. (2015). Origin and distribution of polycyclic aromatic hydrocarbons in recent sediments of El Hito lake (central Spain). Geogaceta 57 (2015), 127-130.

Schorcht F., Kourti I., Scarlet B.M., Roudier S. y Sancho L.D. (2013). Best available techniques (BAT) reference document for the production of cement, lime and magnesium oxide. European Commission, Joint Research Centre, Sevilla, España, 506 pp.

Siti-Aktar I. y Haslenda H. (2015). Low carbon measures for cement plant - A review. J. Clean. Prod. 103, 260-
274. https://doi.org/10.1016/j.jclepro.2014.11.003

Syamala-Devi K., Vijaya-Lakshmi V. y Alakanandana A. (2017). Impacts of cement industry on environment An overview. Asia Pac. J. Tour. Res. 1 (57), 156-161. UNE (2008). Una Norma Española UNE-EN 15259. Calidad del aire. Emisiones de fuentes estacionarias. Requisitos de las secciones y sistios de medición y para el objetivo, plan e informe de medición. Asociación Española de Normalización, 28 de mayo.

UNE (2012). Una Norma Española UNE-EN 17020. Evaluación de la conformidad. Requisitos para el funcionamiento de diferentes tipos de organismos que realizan la inspección. Asociación Española de Normalización, 6 de junio.

UNFCCC (2016). Handbook on the Energy Sector Burning fuels. Memoria. United Nations Framework Convention on Climate Change, Marrakech, Marruecos, $43 \mathrm{pp}$.

Valderrama C., Granados R., Cortina J., Gasol C., Guillem M. y Josa A. (2012). Implementation of the best available techniques in cement manufacturing: A life-cycle assessment study. J. Clean. Prod. 25, 60-67. https://doi. org/10.1016/j.jclepro.2011.11.055

Van den Berg M., Birnbaum L.S., Denison M., De Vito M., Farland W., Feeley M., Fiedler H., Hakansson H., Hanberg A., Haws L., Rose M., Safe S., Schrenk D., Tohyama C., Tritscher A., Tuomisto J., Tysklind M., Walker N. y Peterson R.E. (2006). The 2005 World Health Organization reevaluation of human and mammalian toxic equivalency factors for dioxins and dioxin-like compounds. Toxicol. Sci. 93(2): 223-241. https://doi.org/10.1093/toxsci/kfl055

VDZ (2010). Environmental data of the German cement industry. Manual. Verein Deutscher Zementwerke, Alemania.

Yeo Z., Ng R. y Song B. (2016). Technique for quantification of embodied carbon footprint of construction projects using probabilistic emission factor estimators. J. Clean. Prod. 119, 135-151. https://doi.org/10.1016/j. jclepro.2016.01.076

Zemba S., Ames M., Green L., Botelho M.J., Gossman D., Linkov I. y Palma-Oliveira J. (2011). Emissions of metals and polychlorinated dibenzo(p)dioxins and furans (PCDD/Fs) from Portland cement manufacturing plants: Inter-kiln variability and dependence on fuel types. Sci. Total Environ. 409 (20), 4198-4205. https:// doi.org/10.1016/j.scitotenv.2011.06.047

Zimwara L. y Mugwagwa-Chikowore T.R. (2012). Air pollution control techniques for the cement manufacturing industry: A case study for Zimbabwe. CIE42 Proceedings, Cape Town, South Africa, 16-18 July. 